\title{
Observation and analysis of long-periodic modes in a confluence with dominant tributary inflow
}

\author{
Laurent Schindfessel ${ }^{1,}$, Tom De Mulder ${ }^{1}$, and Mia Loccufier $^{2}$ \\ ${ }^{1}$ Hydraulics Laboratory, Department of Civil Engineering, Ghent University, Ghent, Belgium \\ ${ }^{2}$ Department of Electrical Energy, Metals, Mechanical Constructions and Systems, Ghent University, \\ Ghent, Belgium
}

\begin{abstract}
Confluences with dominant tributary inflow are found to exhibit long-periodic alternations of the flow patterns. They are shown to exist both in laboratory experiments and in numerical simulations. By means of a modal decomposition, insight is given into these long-periodic oscillations. The origin of these oscillations is investigated and their significant influence on the secondary flow patterns in the downstream channel is revealed.
\end{abstract}

\section{Introduction}

Open channel confluences determine the water stages and the transport of sediment and solutes in a hydraulic network of rivers or canals. The characteristics of the confluence hydrodynamics zone are often studied in schematized confluences. The flow features of such a right-angled, asymmetric confluence are depicted in Fig. 1. The key parameter of influence is the discharge ratio $q$, defined as the ratio of main channel discharge to downstream discharge (Fig. 1):

$$
q=Q_{\mathrm{m}} / Q_{\mathrm{d}}=Q_{\mathrm{m}} /\left(Q_{\mathrm{m}}+Q_{\mathrm{t}}\right)
$$

In such a confluence, two shear layers can be found (Fig. 1): the so-called mixing layer between the incoming flows and a shear layer between the separation zone and the merging flows [1-5]. The latter may be absent if no separation zone is formed, which can occur when the downstream corner is rounded, allowing a smooth turning of the tributary flow. In the vicinity of the shear layers, coherent structures are formed by the velocity differences over the shear layers. Examples of such coherent structures are vortices and helical cells (also called streamwise oriented vortical cells).

Vortices can be shed from the respective corners at which either of the shear layers originates. These vortices can be of two types: Kelvin-Helmholtz vortices with fixed rotation sense or wake vortices with alternating rotational sense $[3,6]$. The velocity difference over the shear layer, determined by the discharge ratio $q$, determines which type appears [3].

\footnotetext{
*Corresponding author: laurent.schindfessel@ugent.be
} 
Helical cells appear in confluences because of the curvature of the flow [11]. These cells transport fluid vertically and laterally. Their number and position depend on the flow conditions, the geometry and the bathymetry of the confluence [2-3,8]. Constantinescu et al. [3] discerned that these helical cells can be subjected to large-scale oscillations, which are also influenced by the discharge ratio.

In a laboratory confluence with dominant tributary inflow, i.e. very small discharge ratio $q$, Schindfessel et al. [9] noticed alternations in the surface flow near the right bank, which appeared with an unexpectedly long return period. The presence of this intermittent flow feature was shown by using experimental point velocities and instantaneous velocity fields provided by numerical simulation. However, a deeper analysis to the origin of this phenomenon necessitates advanced processing methods.

In this paper, the cause of the long-periodic modes will be analysed by means of a modal decomposition technique. This type of techniques allows to extract spatial modes from a dataset. Hence, it becomes possible to investigate the full spatial extent of the longperiodic oscillation. The results of this paper are useful for the understanding of coherent structures in confluences.

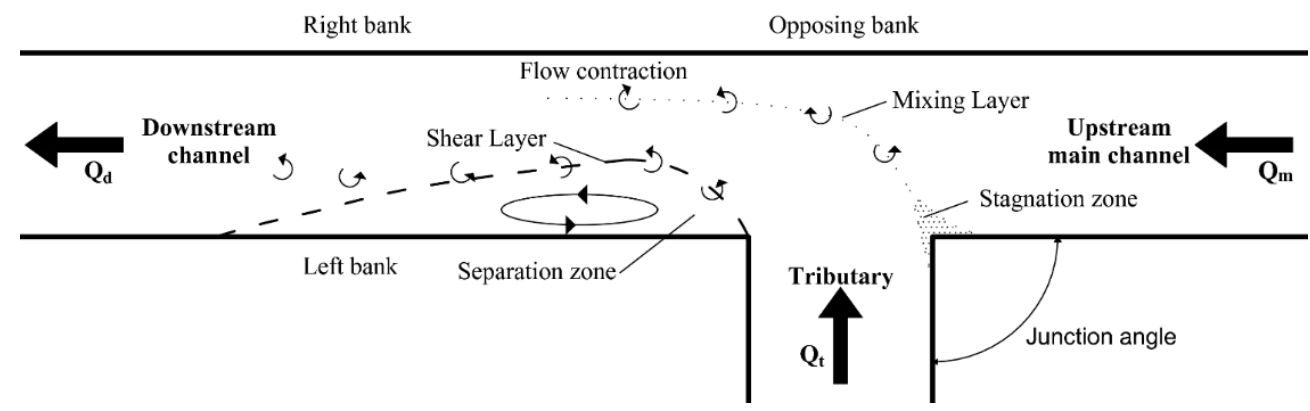

Fig. 1. Conceptual model of an open channel asymmetrical confluence, with a tributary at right angles to a straight channel, and its main flow features (after [1]).

\section{Methodology}

\subsection{Experiments and numerical simulations}

The experiments are conducted in an existing flume with a $90^{\circ}$ confluence angle, similar to the one depicted in Fig. 1. The joining channels have fixed, horizontal and concordant beds. The channels have a chamfered rectangular cross section and an equal top width $W$. For a complete overview of the experimental setup, the reader is referred to [10].

In the experiments, two discharge ratios are tested: $q=0.25$ and $q=0.05$. The first discharge ratio corresponds to a value that is well studied in the literature, whereas the latter corresponds to dominant tributary inflow. The downstream Froude number $(0.05)$ and the downstream water depth $h(0.415 \mathrm{~m})$ are kept constant. As a consequence, the Reynolds number (based on the hydraulic radius) in the downstream channel $\left(98.10^{3}\right)$ is constant too. The surface velocities in the confluence are measured by means of surface particle tracking velocimetry (surface PTV). Additionally, a Vectrino Profiler is used to obtain velocity measurements in the bulk of the flow.

A right-handed coordinate system is adopted, with origin at the bed in the upstream confluence corner. The $x$-axis points in the upstream direction of the upstream main channel, while the y-axis points towards the bank opposing the tributary. The z-axis points vertically upward. To present results in a non-dimensional way, the bulk velocity in the 
downstream channel $U_{\mathrm{d}}$ is used as a velocity scale and the channel width $W$ or downstream depth $h$ are used as a length scale.

Complementary to the experiments, numerical simulations are performed for the aforementioned discharge ratios. The numerical simulations are Large-Eddy Simulations (LES), with the near-wall flow being modelled by a three-layer wall function that includes the effect of the wall roughness (see [10]). The numerical domain contains 4.2 million cells, with $106 \times 37$ cells in the lateral and vertical direction respectively of a cross section. At the surface, a horizontal rigid lid is adopted. The simulations are run for a first period of $760 \mathrm{~s}$, which is long enough to leave the initial transient regime. Then, 1640 more seconds are simulated for data collection [10].

As an inlet condition, a cyclic precursor channel is used. As the cyclic inflow induces a periodicity, the length of this precursor channel was carefully selected to avoid interference with the periodic phenomena that are studied. The inlet conditions induced a periodicity of $0.022 \mathrm{~Hz}$ and $0.065 \mathrm{~Hz}$ in the case of $q=0.25$ and $0.082 \mathrm{~Hz}$ in the case $q=0.05$. For $q=0.05$, random noise superposed on a mean value was used as the main channel inlet, because the low velocities would cause a periodicity with a low frequency, interfering with the longperiodic frequencies under study. Hence, the applied methodology avoids influence of the periodic inlet condition on the low-frequency energy in the spectrum as much as possible.

\subsection{Modal decomposition technique}

Modal decomposition techniques offer the advantage that temporal information can be linked with spatial patterns. Different modal decomposition techniques exist, yet for the present application, a technique is preferred that offers a clear link between mode and frequency. Dynamic Mode Decomposition (DMD) was investigated as a potentially suitable tool, but its capability of discerning the growth rate of modes is dispensable, even adverse for steady state application. Additionally, ranking modes on their importance is not trivial for DMD. Ultimately, Discrete Fourier Transform (DFT) was selected as decomposition tool, as it combines a clear relation between mode and frequency with a more robust identification of important modes. The mentioned DFT is a Fourier Decomposition of the velocity field (vector field) into a set of spatial modes (vectors) that oscillate with a fixed frequency, which Basley [11] refers to as global Fourier decomposition.

To calculate the DFT of the velocity fields, the modred package [12] is used, utilising the property that a DFT is equal to a DMD analysis of mean-subtracted data [13]. The whole time series of collected data is decomposed, with a sampling frequency of $1 \mathrm{~Hz}$. The DFT analysis is limited to an area around the mixing layer, over the entire depth of the flow. The investigated area corresponds to the plotted area in results figures, e.g. in Fig. 6.

\section{Experimental observations}

Long-periodic oscillations are observed in the laboratory experiments, yet only for the case $q=0.05$. Figure 2 illustrates this by presenting the surface PTV experiments at two characteristic time instances. At one instance in time, the flow in the confluence appears as expected based on conceptual models (Fig. 2(a)): the flow from the main channel (in the top of the image) is deflected by the tributary inflow (from the right) as it enters the region of flow contraction. At other instances in time, all particles that come from the main channel migrate to the right and coalesce in the mixing layer (Fig. 2(b)). This movement is driven by the upwelling of fluid near the right bank (on the left of the images, opposite to the tributary channel). Figure 2(b) presents the onset of this movement, where some particles on the main channel's side of the mixing layer are pushed towards the mixing 
layer. At later times, the particles in the mixing layer agglomerate even more, when the mixing layer is pushed further to the tributary's side. The mixing layer is sometimes pushed so far that it enters the downstream channel at half the downstream channel's width. These two flow modes can be present during several minutes, yet alternate. There is thus an unexpectedly long return period of these flow oscillations.

To quantify the flow modes, ADV measurements with a duration of 30 min are performed in the point $(x / W=-1.33, y / W=0.75, z / h=0.95)$. This point is close to the right bank and the surface, where the shifts in the position of the mixing layer are observed. Following the methodology used by Constantinescu et al. [12], histograms of lateral velocity are analysed. Under ordinary conditions, the histograms are expected to show a single peak, whose width is related to the strength of turbulent motions. If the histograms exhibit two peaks, this is a very strong indication that two flow equilibria are present, a phenomenon called bimodal oscillations by Constantinescu et al.[12].

In the $q=0.25$ case, the experimental lateral velocities show a sharp histogram with a nearly symmetrical distribution around the mean (Fig. 3(a)). Yet, in the $q=0.05$ case, the histogram does not have a Gaussian shape. It resembles more the summation of two Gaussian shapes, with means around $-0.5 U_{\mathrm{d}}$ and $0.2 U_{\mathrm{d}}$ (Fig. 3(b)). Hence, there are two flow conditions that differ in the direction of the lateral velocity near the right bank. This shows that there is a new flow feature appearing when the discharge ratio is small.

To exclude a time-varying influence from upstream or downstream boundary conditions, long term ADV measurements were made at locations far upstream and downstream of the junction. Yet, no fluctuations were found that might explain the observed intermittent behaviour in the junction region. Therefore, the intermittent behaviour seems to be a distinct confluence-induced flow feature.

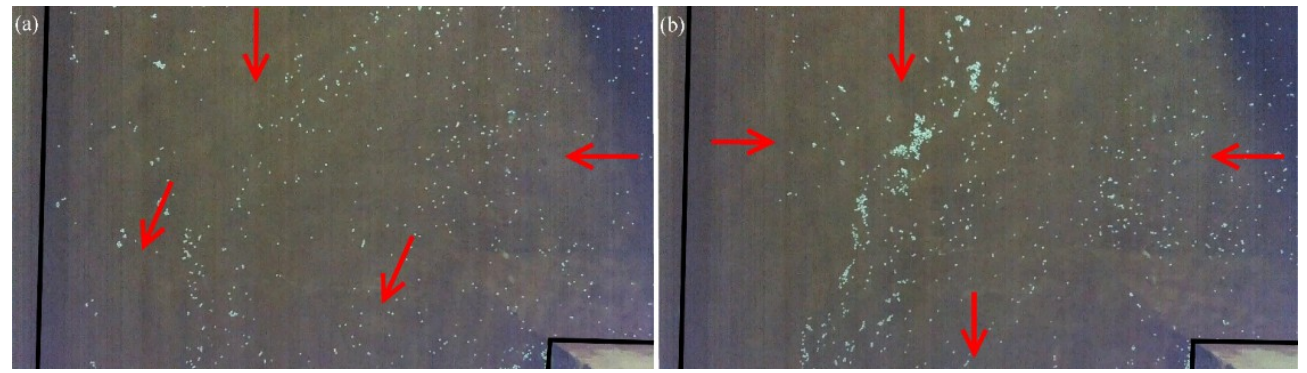

Fig. 2. Two characteristic time instances during the surface PTV experiments of the $q=0.05$ case: (a) flow corresponding to the conceptual model; (b) the onset of upwelling flow. The black lines indicate the walls of the flume and the arrows illustrate the flow direction. The downstream confluence corner can be found in the bottom right of each picture. See the online version of a colour image.
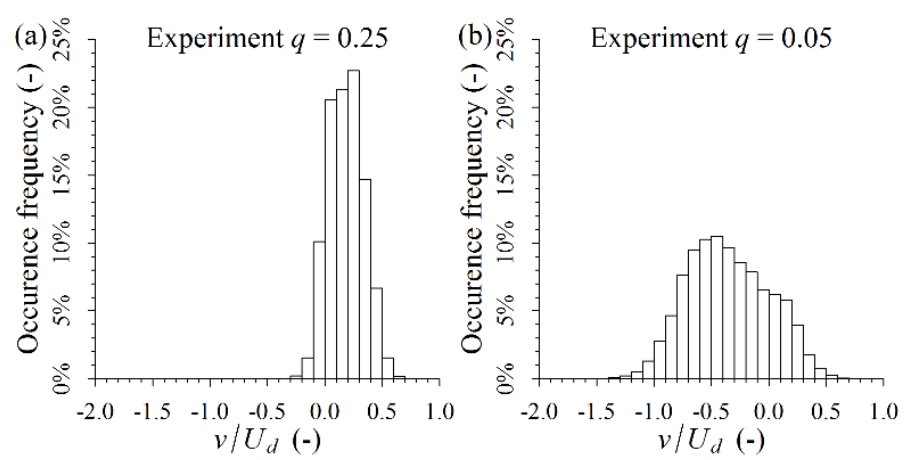

Fig. 3. Histogram of the lateral velocity $v$ in the point $(x / W=-1.33, y / W=0.75, z / h=0.95)$ during a 30 min long measurement by means of the ADV for the discharge ratio (a) $q=0.25$ and (b) $q=0.05$. 


\section{Numerical observations and analysis with modal decomposition techniques}

In the simulation of $q=0.05$, there are also oscillations in the lateral velocity of the same point as measured in the experiments, although their return period is much smaller in the numerical simulations than in the experiment (not shown). Despite the different return period, the finding that these unexpected changes are present in both the experiment and the numerical model suggests that they are not a measuring artefact.

The DFT is applied to the simulated, instantaneous (spatial) velocity fields. The resulting modes represent flow oscillations over the entire processed area. It should be noted that DFT is actually derived for periodic oscillations, whereas the present numerical simulations show rather aperiodic oscillations. Therefore, the DFT might show a smeared instead of a sharp frequency peak.

The spectrum of the two simulations is shown in Fig. 4, for the case $q=0.05$. The spectrum of the shortened precursor simulation shows some peaks at very low frequencies (indicated with arrows in Fig. 4), namely at $0.0018 \mathrm{~Hz}$ (mode M1) and $0.0037 \mathrm{~Hz}$ (M2). The latter is the higher harmonic of the former. Additionally, a peak around $0.081 \mathrm{~Hz}(\mathrm{M} 3)$ is present, coinciding with the frequency induced by the cyclic tributary channel $(0.082$ $\mathrm{Hz}$ ). So the simulations reveal that low frequency modes dominate the spectrum at $q=0.05$ and that no other modes of substantial energy are present (Fig. 4). This means that vortex shedding is not important in this case, which is attributed to the curvature of the mixing layer with the largest velocities on the inner side [14].

Mode M1 has a very low frequency $(0.0018 \mathrm{~Hz}$, corresponding to a period of $556 \mathrm{~s})$ of similar order as the return period of the intermittent flow features. For this reason, it is extensively visualized. As a start, Fig. 5 shows the velocity field and the streamwise oriented vorticity $\omega_{\mathrm{s}}$ at a height of $z / h=0.8$. The velocities in the section $x / W=-0.25$ (Fig. 6) are also provided. From all figures, it follows that the velocity near the surface of the downstream channel is very strong. Particularly, it is the lateral component that alternates between negative and positive values. This agrees well with the observations of the intermittency in the laboratory experiments (Fig. 2). Moreover, the amplitude of this mode is so strong that it can explain the shift in lateral velocity noticed in the numerical simulations. It is thus concluded that the selected mode is the DFT representation of the intermittent flow feature.

The DFT decomposition offers the advantage that all flow oscillations appearing with the same frequency are contained in the mode. Hence, analysis of the selected mode gives further insight in the nature of the intermittent flow features. For example, Fig. 5 demonstrates that the alternating lateral velocities are associated to a helical cell appearing around $y / W=0.75$. The amplitude of this cyclic cell is of the same order as the timeaveraged value (not shown), which indicates that in the simulation, there was alternately a clockwise and a counter-clockwise streamwise oriented vortex. The section $x / W=-0.25$ is presented to illustrate how this streamwise oriented vortex emerges (Fig. 6). It shows that the tributary flow can breach into the confluence area near the surface (Fig. 6(a)), $z / W \approx$ 0.3 ). Once the tributary flow impinges on the opposite wall, a positive helical cell is created near the top and surface velocities are directed towards the right bank in the downstream channel (Fig. 6(b)). Later, the tributary intrudes the confluence area close to the bottom (Fig. 6(c)) leading to a cell of opposite rotation when the flow impinges on the opposing wall (Fig. $6(\mathrm{~d})), z / W \approx 0.1$ ). The fluctuations in the position of the tributary's inflow are located near the upstream junction corner and precede the velocity fluctuations in the downstream channel (Fig. 5). Consequently, it is the depth at which the tributary flow enters the confluence area that determines whether the tributary flow impinges on the opposing bank near the surface or near the bed. This subsequently defines the rotational 
sense of the helical cells in the downstream channel. Alternations of the intrusion depth form the origin of the observed flow pattern.

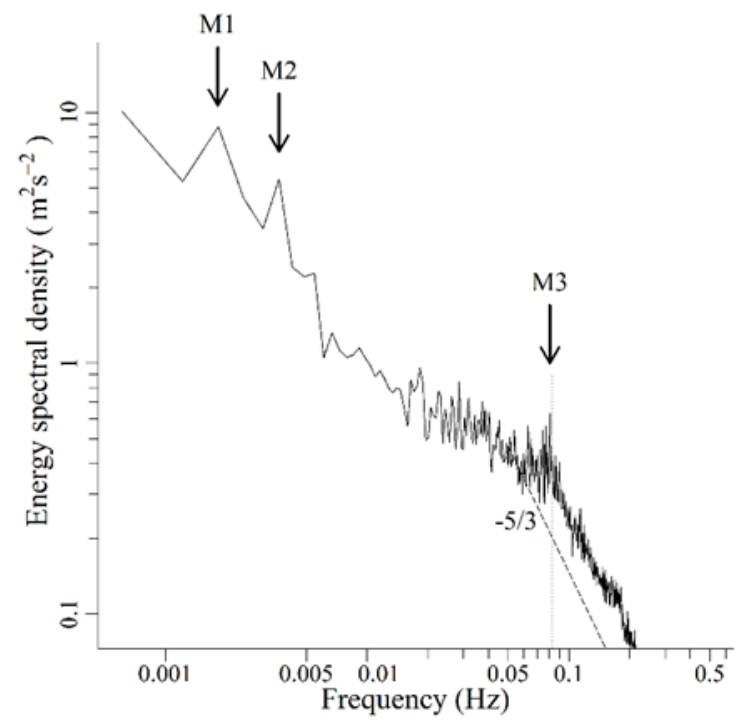

Fig. 4. Energy spectral density against frequency in the mixing layer of the case $q=0.05$. The vertical dotted lines indicate the frequency imposed by the cyclic precursor in the tributary channel, while the arrows indicate the identified frequency peaks.

\section{Discussion}

The intermittent behaviour changes the confluence flow considerably. It is natural to call the intermittent phenomenon a large-scale structure or bi-modal oscillation, following the terminology of Constantinescu et al. [2]. However, this terminology was used for oscillations of helical cells in the mixing layer, whereas the present observation originates from shifts in the height above the bed at which the tributary flow breaches into the main flow and at which it subsequently impinges on the opposite bank. To keep this distinction in the terminology, the present observation will be named "intermittent flow feature".

The intermittent flow feature is a phenomenon that appears at small q. When the simulation of the case $q=0.05$ is run during a time interval that is more than three times larger than the original simulation, the intermittent flow feature continues to exist in the simulation, although no fixed return period seems to be present (not shown). Despite this fact, DFT was able to identify the motion at a single frequency, which is the frequency that matches best to the analysed time series. Note, however, that the return period in the numerical simulations is known to differ from the experimental one.

Although the intermittent flow feature originates near the upstream junction corner, it has a huge impact on the flow in the downstream channel because of the presence of the opposing bank. The impinging of the tributary flow on this bank induces namely alternating helical flow in the downstream channel. Helical flow can form a means of sediment transport and can cause an increase in bed shear [2]. However, the use of schematized cross-sectional shapes and a $90^{\circ}$ junction angle may be beneficial for the emergence of the intermittent flow feature. It can thus be argued that a smaller confluence angle or wider main channel may disfavour the occurrence of this phenomenon. Larger Froude numbers, on the other hand, may aid the development of the intermittent flow feature, since more momentum will be present in the tributary channel. 

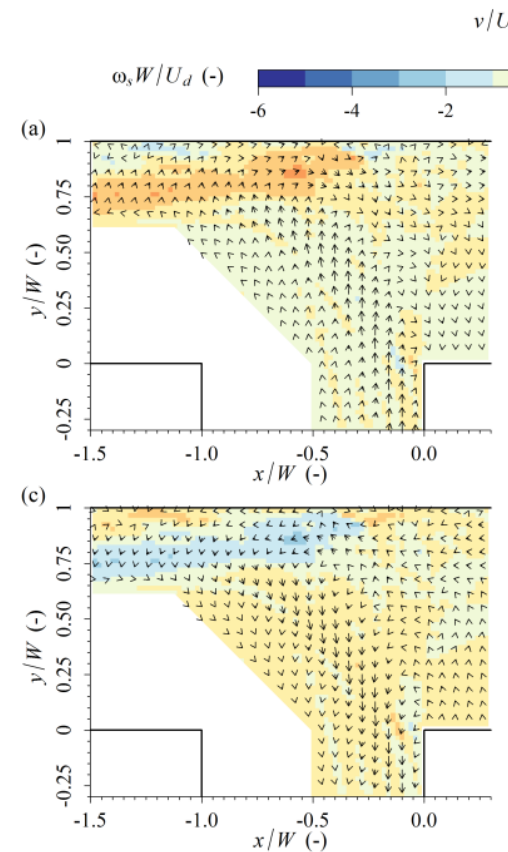

$\begin{aligned} v / U_{d} & =0.2(-)\end{aligned}$
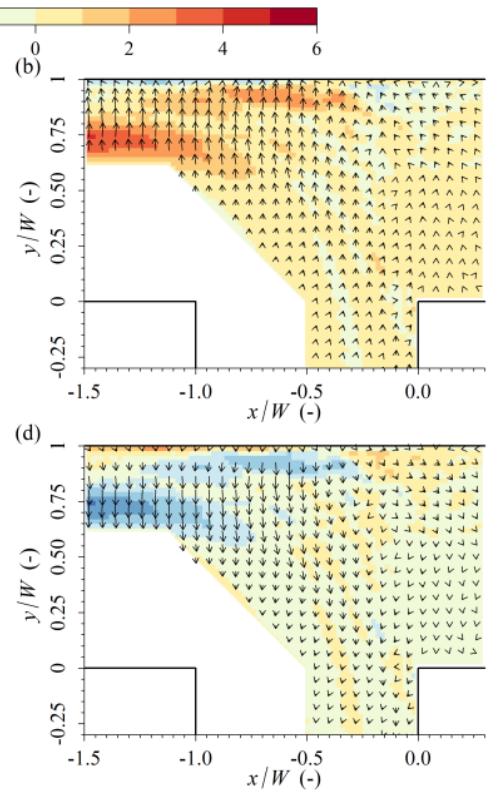

Fig. 5. Mode $\mathrm{M} 1$ in the mixing layer of $q=0.05$ : horizontal section at $z / h=0.8$. Colour scale represents $\omega_{\mathrm{s}}$, whereas arrows represent in-plane velocity; (a) $1 / 4 T$; (b) $1 / 2 T$; (c) $3 / 4 T$ and (d) $T$, with $T$ representing the DFT period. See the online version of a colour image.

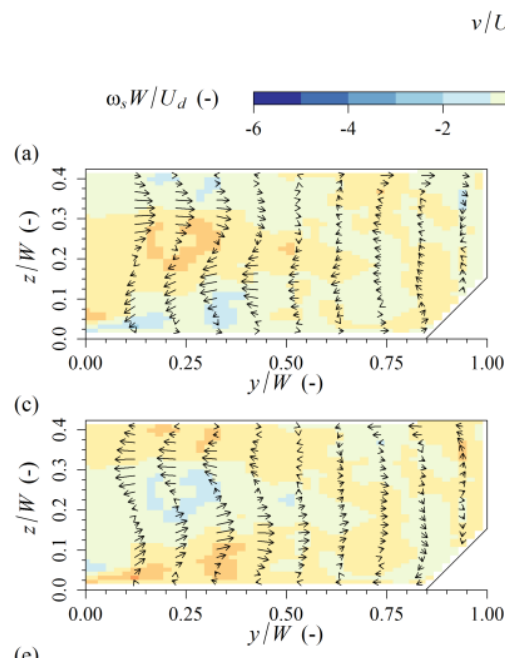

$$
v / U_{d}=0.2(-)
$$

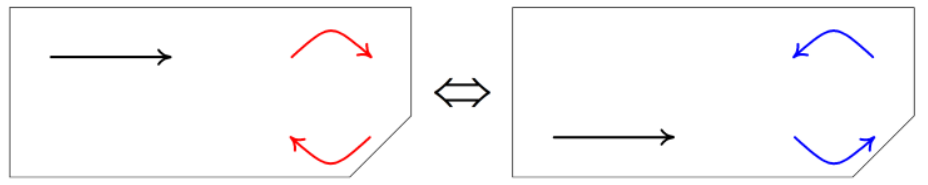

Fig. 6. Mode $\mathrm{M} 1$ in the mixing layer of $\mathrm{q}=0.05$ : vertical section at $\mathrm{x} / \mathrm{W}=-0.25$. Colour scale represents $\omega_{\mathrm{s}}$, whereas arrows represent in-plane velocity; (a) $1 / 4 T$; (b) $1 / 2 T$; (c) $3 / 4 T$ and (d) $T$, with $T$ representing the DFT period. (e) presents a schematic sketch of the mode. See the online version of a colour image. 


\section{Conclusion}

Long-periodic oscillations of the flow were observed in confluences of open channels with dominant tributary inflow, both in lab experiments and in numerical simulations. By means of a modal decomposition technique, it was shown that the depth at which the tributary flow enters the confluence can alternate. As a consequence, also the impingement of the (dominant) tributary flow on the opposing wall can alter between locations close to the surface and near the bed, causing a different type of secondary flow in the downstream channel. The return period of these alternations was longer than would be expected. For confluences with dominant tributary flow, the flow can thus be subjected to intermittent behaviour.

The authors would like to acknowledge the funding that the first author received as Ph.D. fellow of the Research Foundation - Flanders (FWO). The computational resources and services used in this work were provided by the VSC (Flemish Supercomputer Center).

\section{References}

1. J. L. Best, Flow dynamics and sediment transport at river channel confluences, Ph.D. thesis, Univ. London (1985)

2. G. Constantinescu, S. Miyawaki, B. L. Rhoads, A. N. Sukhodolov, G. Kirkil, Structure of turbulent flow at a river confluence with momentum and velocity ratios close to 1 : Insight provided by an eddy-resolving numerical simulation, W.R.R., 47, 5 (2011)

3. G. Constantinescu, S. Miyawaki, B. L. Rhoads, A. N. Sukhodolov, Numerical analysis of the effect of momentum ratio on the dynamics and sediment-entrainment capacity of coheren flow structures at a stream confluence, J.G.R.: E.S., 117 (2012)

4. E. Mignot, I. Vinkovic, D. Doppler, N. Riviere, Mixing layer in open-channel junction flows, E.F.M., 14, 5 (2014)

5. S. Guillén Ludeña, Z. Cheng, G. Constantinescu, M. J. Franca, Hydrodynamics of mountain-river confluences and its relationship to sediment transport, J.G.R.: E.S., 122, 4 (2017)

6. K. M. Konsoer, B. L. Rhoads, Spatial-temporal structure of mixing interface turbulence at two large river confluences, E.F.M., 14, 5 (2014)

7. B. L. Rhoads, S. T. Kenworthy, Flow structure at an asymmetrical stream confluence, Geom., 11, 4 (1995)

8. B. L. Rhoads, A. N. Sukhodolov, Spatial and temporal structure of shear layer turbulence at a stream confluence, W.R.R., 40, 6 (2001)

9. L. Schindfessel, S. Creëlle, T. De Mulder, Flow patterns in an open channel confluence with increasingly dominant tributary inflow, Water, 7, 9 (2015)

10. L. Schindfessel, Numerical and experimental modelling of the hydrodynamics of open channel confluences with dominant tributary inflow, Ph.D. thesis, Ghent Univ. (2007)

11. J. Basley, An experimental investigation on waves and coherent structures in a threedimensional open cavity flow, Ph.D. thesis, Univ. Paris-Sud (2012)

12. B. A. Belson, J. H. Tu, C. W. Rowley, Algorithm 945: Modred: A parallelized model reduction library, ACM Trans. Math. Softw. 40, 4 (2014)

13. K. K. Chen, J. H. Tu, C. W. Rowley, Variants of dynamic mode decomposition: Boundary condition, koopman, and fourier analyses, J. Nonl. Sc., 22, 6 (2012)

14. C. Wang, The effects of curvature on turbulent mixing layers, Ph.D. thesis, CIT (1984) 